\title{
Human Parechovirus Genotypes -10, -13 and -15 in Pakistani Children with Acute Dehydrating Gastroenteritis
}

\author{
Muhammad Masroor Alam ${ }^{1}$, Adnan Khurshid ${ }^{2}$, Shahzad Shaukat ${ }^{2}$, Muhammad Suleman Rana ${ }^{2}$, \\ Salmaan Sharif ${ }^{2}$, Mehar Angez ${ }^{2}$, Nadia Nisar ${ }^{2}$, Muhammad Naeem ${ }^{1}$, Syed Sohail Zahoor Zaidi ${ }^{2 *}$ \\ 1 Department of Biotechnology, Quaid-i-Azam University, Islamabad, Pakistan, 2 Department of Virology, National Institute of Health, Islamabad, Pakistan
}

\begin{abstract}
Human parechoviruses are known to cause asymptomatic to severe clinical illness predominantly respiratory and gastroenetric infections. Despite their global prevalence, epidemiological studies have not been performed in Pakistan. In this study, we retrospectively analyzed 110 fecal specimen and found 26 (24\%) positive for viral RNA with HPeV-10 ( $n=3$, $23 \%), \mathrm{HPeV}-13(n=4,31 \%)$ and HPeV-15 $(n=6,46 \%)$ genotypes. Clinical features of patients with different HPeV genotypes were compared. All HPeV positive children were aged $\leq 4$ years (mean 13.92 months). The male-to-female ratio was 1: 1.17 (46.2 vs $53.8 \%)$ with significant association $(p=.031)$ to HPeV infectivity. HPeV-10 and -13 were found during summer while $\mathrm{HPeV}-15$ was only detected during late winter season. Disease symptoms were more severe in children infected with HPeV-10 and -13 as compared to HPeV-15. Fever and vomiting were observed in $100 \%$ cases of HPeV-10 and -13 while only $17 \%$ patients of HPeV-15 had these complaints. Phylogenetic analyses showed that HPeV-10, -13 and -15 strains found in this study have $9-13 \%, 16.8 \%$ and $21.8 \%$ nucleotide divergence respectively from the prototype strains and were clustered to distinct genetic lineages. This is the first report of HPeV-15 infection in humans although first identified in rhesus macaques. The arginine-glycine-aspartic acid (RGD) motif present at the C-terminal of VP1 responsible for the viral attachment to cellular integrins was not found in all of these strains. In conclusion, these findings enhance our knowledge related to the epidemiology and genetic diversity of the HPeV in Pakistan and support the need for continued laboratory based surveillance programs especially in infants and neonatal clinical settings. Further, the parechovirus pathogenesis, cross-species transmission and disease reservoirs must be ascertained to adopt better prevention measures.
\end{abstract}

Citation: Alam MM, Khurshid A, Shaukat S, Rana MS, Sharif S, et al. (2013) Human Parechovirus Genotypes $-10,-13$ and -15 in Pakistani Children with Acute Dehydrating Gastroenteritis. PLoS ONE 8(11): e78377. doi:10.1371/journal.pone.0078377

Editor: Jianming Qiu, University of Kansas Medical Center, United States of America

Received July 25, 2013; Accepted September 19, 2013; Published November 12, 2013

Copyright: (C) 2013 Alam et al. This is an open-access article distributed under the terms of the Creative Commons Attribution License, which permits unrestricted use, distribution, and reproduction in any medium, provided the original author and source are credited.

Funding: No current external funding sources for this study.

Competing Interests: The authors have declared that no competing interests exist.

*E-mail: zaidis@pak.emro.who.int

\section{Introduction}

Human Parechovirus belongs to family picornaviridae within the genus parechovirus and contains a single stranded RNA genome encapsidated in a non-enveloped icosahedral capsid protein coat. The prototypes of this virus, $\mathrm{HPeV}-1$ and $\mathrm{HPeV}-2$, were initially known as echo-22 and echo-23, under the genus enterovirus but were re-classified into a separate genus on the basis of their genome organization and biological properties [1,2]. Since their discovery in 1956 [3], several new genotypes have been identified within the past few years. To date, there have been 16 reported $\mathrm{HPeV}$ genotypes (www.picornaviridae.com/parechovirus/hpev/ hpev.htm) although published data is available for only 12 genotypes 1-8, 10, 11, 14 and 12 [4].

$\mathrm{HPeVs}$ have been recognized as an important pediatric viral pathogen causing mild to severe infections including gastroenteritis, respiratory infections, encephalitis and flaccid paralysis in children under 5 years of age $[5,6]$. HPeV-1 is often isolated from children with diarrhea and gastroenteritis [7]. HPeV-3 has been found associated with transient paralysis, diarrhea [7] and neonatal sepsis [8]. HPeV-4 was isolated from a 5 year old patient with lymphadenitis [9]. $\mathrm{HPeV}-6$ was identified in a 1 year old girl with Reye syndrome [10]. HPeV-7 was isolated from a patient with non-polio acute flaccid paralysis in Pakistan [11]. $\mathrm{HPeV}-8$ was identified in a patient with enteritis in Salvador, Brazil [11]. $\mathrm{HPeV}-10$ and -11 were identified in patients with acute gastroenteritis in Sri Lanka $[12,13]$. HPeV-12 was detected in 20 months old child with diarrhea and paralysis [4] while HPeV-14 was detected from a febrile child in the Netherlands [14].

The studies about the spectrum of causative agents causing acute gastroenteritis have never been conducted in Pakistan except few focused only on rotavirus in the recent years [15-17]. Human Parechovirus genotypes $-1,-5,-6$ and -7 have been previously identified from acute flaccid paralysis cases in Pakistan through metagenomic approach while HPeV-12 was recently identified in a Pakistani child with diarrhea and paralysis $[4,11]$. To the best of our knowledge, comprehensive epidemiological studies focused on the prevalence of human parechovirus in hospitalized children presented with acute gastroenteritis have never been conducted in Pakistan. We here report the epidemiological and virological information obtained from retrospective analysis of stool samples collected during 2008. Due to their possible role in gastroenteritis, the molecular properties and genetic diversity of parechovirus strains were studied in relation to their clinical significance. 


\section{Materials and Methods}

In this study, stool samples from 110 children below five years were collected during January to December 2008. The children were hospitalized if fulfilled the case definition criteria of gastroenteritis. The case definition of gastroenteritis was $\geq 3$ loose stools in 24 hours, vomiting $\geq 3$ times in 24 hours, loose stools with two additional symptoms or vomiting with two additional symptoms. Additional symptoms included diarrhea, vomiting, nausea, fever, abdominal pain, abdominal cramps, and blood or mucus in stool. The study concept and design was approved through the Pakistan's National Institute of Health Internal Review Board. All samples were collected after informed and written consent from the patient's parents/guardians at the time of sample collected on a standard questionnaire approved by the NIH Internal review board.

The collected samples were analyzed for the presence of $\mathrm{HPeV}$ RNA directly by real time PCR as described earlier [18]. Although this 5'UTR based real-time assay detects both HPeV and Ljungan virus RNA, HPeV was confirmed by amplification of $760 \mathrm{bp}$ fragment within VPl gene positioned from nt. 2332 to 3090 according to the prototype strain Harris (GenBank accession number L02971) using the primers VP1-parEchoFl (5'CGAAAATTCRTGGGGTTC-3') and VP1-parEchoR1 (5'AAACGYCTRTCTAAATAWGG-3') as described by Benschop et al [19]. The PCR amplicons of VPl gene were purified and sequenced in both directions using a BigDye Terminator cycle sequencing kit v3.1 (Perkin Elmer-Applied Biosystems, Inc., Foster City, CA). The sequence data was collected by an ABI Prism genetic analyzer 3100, edited using Sequencher v.4.1 (Gene Codes Inc., Ann Arbor, MI, USA) and the phylogenetic tree was reconstructed using MEGA 4.0.

$\mathrm{HPeV}$ genotypes were determined on the basis of sequence analysis of VPl gene fragment with threshold of $\geq 77 \%$ and $\geq 87 \%$ nucleotide and amino acid identity respectively [20]. Phylogenetic analyses were performed in comparison to the strains belonging to different genotypes and geographical regions as retrieved from GenBank. Evolutionary tree and distances (number of base substitutions per site) were generated by Neighbor Joining method with Kimura-2 parameter using MEGA 4.0 (http:// megasoftware.net/). The percentage of replicate trees in which the associated taxa clustered together in the bootstrap test (1000 replicates) is shown next to the branches. The GenBank accession numbers, country, year of sample collection and respective genotype information has been given where available. The HPev strains found in this study have been submitted to GenBank under the accession Nos. KF626450-62.

The descriptive statistical analysis like frequencies, percentages, ratio, mean, median and range were carried out for age, gender, duration of symptoms and treatment. The analysis of variance (ANOVA) was performed for the comparison of various demographic features among $\mathrm{HPeV}-10, \mathrm{HPeV}-13$ and $\mathrm{HPeV}-15$ infected patients. The p-value less than 0.05 (two sided) were considered statistically significant. The association between different $\mathrm{HPeV}$ genotypes and disease severity was established through Fisher's Chi-square test $(\chi 2)$. The statistical analyses were performed by using SPSS ver. 18.

\section{Results}

From January-December 2008, a total of 26/110 (24\%) clinical samples were determined to be positive for parechovirus by realtime RT-PCR in our laboratory. Of those, HPeV genotypes were identified in 13/26 (50\%) samples. The majority of the isolates $(n=6)$ could be identified as HPeV-15 $(46 \%)$. In addition, $(n=4)$ isolates were genotyped as $\mathrm{HPeV}-13(31 \%)$ and $(\mathrm{n}=3)$ isolates were identified as $\mathrm{HPeV}-10$ (23\%). Hundred percent of all $\mathrm{HPeV}$ cases reported during this time period were survived after Oral Rehydration therapy (ORT) and/or Intravenous rehydration therapy.

\section{Demographical Features of HPeV positive Cases}

All of the 110 children presented with gastroenteritis were aged $\leq 4$ years (mean 13.92 months; median 8 months; range 1 month to 4 years), whereas the mean age for $\mathrm{HPeV}$ positive cases was $13.9 \pm 13.2$ months (median 8 months; range 1 to 40 months) (Table 1). HPeV-10 and -13 infections were more frequently found in females $(50 \%$ and $100 \%$ respectively) as compared to males; however, $83 \%$ infections with $\mathrm{HPeV}-15$ genotypes were found in males. All the cases with $\mathrm{HPeV}-15$ were identified in winter while infections with $\mathrm{HPeV}-10$ and -13 were reported during the monsoon months of June and July (Figure 1).

The mean duration of $\mathrm{HPeV}$ symptoms was recorded as $2.5 \pm 1.6$ days. Among the $\mathrm{HPeV}$ positive cases, the number of vomiting and diarrhea episodes per 24 hours was found to be $3.5 \pm 3.2$ and $7.8 \pm 1$ respectively. The symptoms of diarrhea persisted for $3 \pm 2.3$ days whereas vomiting continued for $1.8 \pm 2$ days. Upon hospitalization, all of the HPeV infected children were recovered within 1-3 days of oral and/or intravenous rehydration therapy.

\section{Comparison of HPeV Genotypes and Diseases Severity}

Infections with different $\mathrm{HPeV}$ genotypes were compared for disease severity and clinical symptoms using Fisher's Chi-square test $\left(\chi^{2}\right)$ (Table 2). Among the three genotypes, fever, vomiting, diarrhea and ORT treatment were found statistically significant $(p<0.05)$. Disease symptoms were more severe in children infected with $\mathrm{HPeV}-10$ and -13 as compared to $\mathrm{HPeV}-15$. For instance, fever and vomiting were observed in $100 \%$ cases of $\mathrm{HPeV}-10$ and -13 while only $17 \%$ patients of $\mathrm{HPeV}-15$ had these complaints. In

Table 1. Comparative analysis of clinical variables (quantitative) among children positive for human parechovirus (HPeV) infection in Pakistan using ANOVA.

\begin{tabular}{llll}
\hline Characteristics & Mean \pm S.D & Range & p-value \\
\hline Age (months) & $13.9 \pm 13.2$ & $(1-40)$ & 0.345 \\
\hline Duration of Symptoms (days) & 8 & & \\
\hline Vomiting Episodes per 24 hours & $2.5 \pm 1.6$ & $(1-5)$ & $0.016^{*}$ \\
\hline Vomiting Duration (days) & 3 & & \\
\hline Diarrhea episodes per 24 hours & $6.5 \pm 3.2$ & $(0-8)$ & $0.003^{*}$ \\
\hline Diarrhea Duration (days) & $1.8 \pm 2$ & $(0-6)$ & $0.008^{*}$ \\
\hline Duration of Treatment (days) & 2 & & \\
& $8.8 \pm 1$ & $(6-10)$ & $0.019^{*}$ \\
\hline & $2.9 \pm 2.3$ & $(1-9)$ & $0.048^{*}$ \\
\hline
\end{tabular}

Values with Mean $\pm S D$, Median and Range are given for positive cases. The data values with asterisk.

*indicates significant $p$-values.

doi:10.1371/journal.pone.0078377.t001 
\#\#HPeV-10

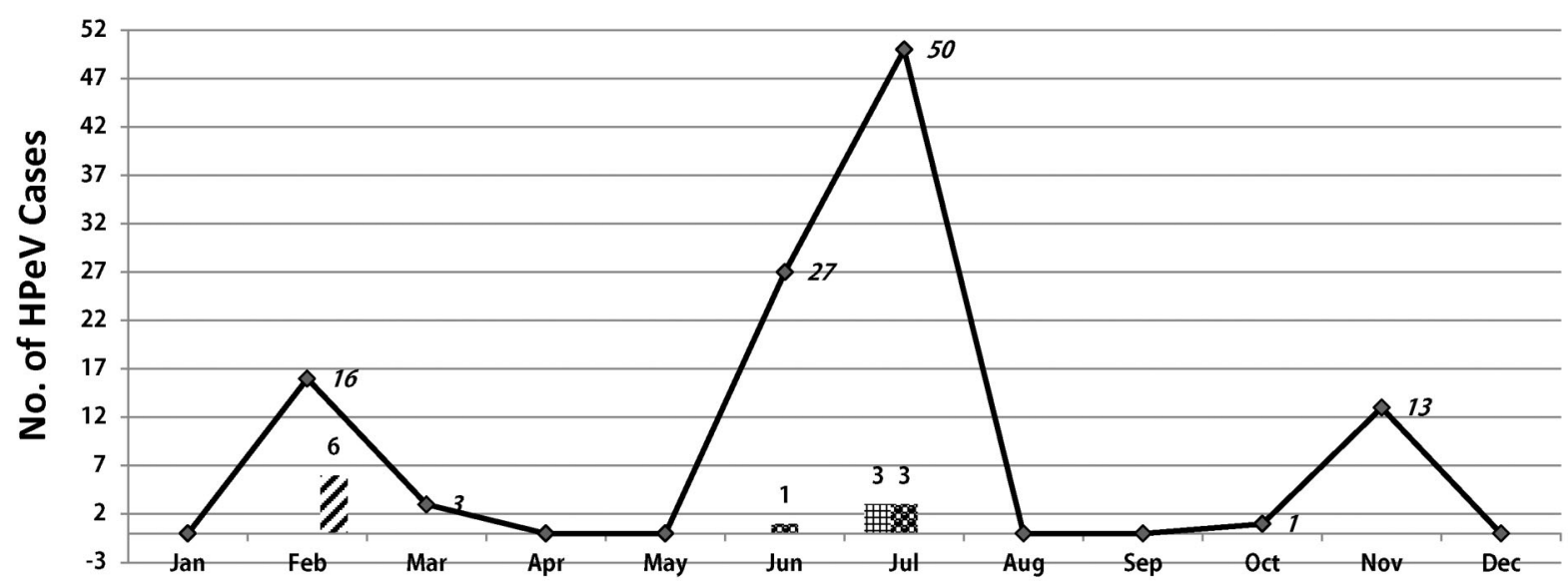

Months-2008

Figure 1. The distribution of HPeV infections in Pakistan during 2008 is presented with months given on X-axis. The data labels on $\mathrm{Y}$ axis indicate the number of total and positive samples for each genotype across the year. Total number of cases is indicated by solid black line indicating typical peak season for gastroenteritis in summer (monsoon/rainy season) months. The number of cases positive for each genotype is indicated with different bar-style.

doi:10.1371/journal.pone.0078377.g001

contrast, all $\mathrm{HPeV}$ infected children, irrespective of the genotype, were presented to the hospital with the complaints of diarrhea although the duration was prolonged among HPeV-13 cases ( $4.75 \pm 3$ days). Similarly, the duration of vomiting remained for $3 \pm 1.48$ days in $\mathrm{HPeV}-10$ and -13 cases as compared to HPeV-15 cases $(0.17 \pm 0.40)$. To conclude, disease symptoms were significantly more severe in $\mathrm{HPeV}-10$ and -13 cases as compared to $\mathrm{HPeV}-15$ cases $(p<0.05)$.

\section{Molecular identification of HPeV}

Thirteen (50\%) out of 26 samples were successfully genotyped by VP1 analysis (Table 2). The majority of isolates were identified as $\mathrm{HPeV}-15(46 \%)$ in addition to HPeV-13 (31\%) and HPeV-10 $(23 \%)$. All children with $\mathrm{HPeV}$ infection were survived (mean treatment days $=2$ ). The mean age of children positive for $\mathrm{HPeV}$ $10, \mathrm{HPeV}-13$ and HPeV-15 was 4.33 months (range $1-7$ months), 14.25 months (range 6-24 months) and 18 months (range $1-40$ months) respectively ( $p=0.345$; Analysis of Variance). All HPeV13 infections were observed in boys while male to female ratio for $\mathrm{HPeV} 10$ and HPeV15 infections were found as 2:1 and 1:5 respectively $(p=0.031)$.

\section{Phylogenetic Analysis}

Nucleotide and deduced aminoacid (aa) identities were compared with the prototype sequences of respective genotypes. For $\mathrm{HPeV}-10$, three strains from this study showed $100 \%$ identity to each other while $87-91 \%$ nucleotide identity was found with the prototype strains from Bangladesh (GenBank Accession No. JX219568). For HPeV-13, we detected four strains in our samples with $16.8 \%$ nucleotide and $5.1 \%$ aminoacid divergence from the prototype strain detected in Bangladesh (GenBank Accession No. JX219579). Similarly, 6 strains of HPeV-15 from Pakistan were $100 \%$ identical with $21.8 \%$ nucleotide divergence from the prototype strain reported from Bangladesh (GenBank Accession No. JX219573). Genetic analysis revealed that all HPeV viruses detected in our samples present distinct lineages than the previously known strains. The prototypes of $\mathrm{HPeV}-13$ and $\mathrm{HPeV}-$ 15 have only been detected in Bangladesh while HPeV-10 has been found in Sri Lanka as well. However, the viruses from each of these geographical regions were placed into separate lineages (Figure 2).

The RGD (Arginine-glycine-aspartic acid) motif present at the $\mathrm{C}$-terminal region of $\mathrm{VP} 1$ gene has been previously detected in few of the $\mathrm{HPeV}$ types but no such motif was found in study strains of $\mathrm{HPeV}-10,-13$ and -15 .

\section{Discussion}

Table 2. Comparison of demographic and clinical details of patients infected with different genotypes of human parechovirus.

\begin{tabular}{llll}
\hline Mean \pm S.D & Range & $p$-value \\
\hline Age (months) & $13.9 \pm 13.2$ & $(1-40)$ & 0.345 \\
Duration of Symptoms (days) & $2.5 \pm 1.6$ & $(1-5)$ & $0.016^{*}$ \\
\hline Vomiting Episodes per 24 hours & 3 & & \\
& $3.5 \pm 3.2$ & $(0-8)$ & $0.003^{*}$ \\
Vomiting Duration (days) & 6 & & \\
& $1.8 \pm 2$ & $(0-6)$ & $0.008^{*}$ \\
Diarrhea episodes per 24 hours & 2 & & \\
& $7.8 \pm 1$ & $(6-10)$ & $0.019^{*}$ \\
Diarrhea Duration (days) & 8 & & \\
\hline Duration of Treatment (days) & $2.9 \pm 2.3$ & $(1-9)$ & $0.048^{*}$ \\
\hline & 3 & & \\
\hline
\end{tabular}

The data values with asterisk.

*indicates significant $p$-values. $\mathrm{M}=$ Male; $\mathrm{F}=$ Female; $\mathrm{ORT}=$ Oral Rehydration Therapy; IV = Intravenous.

doi:10.1371/journal.pone.0078377.t002 


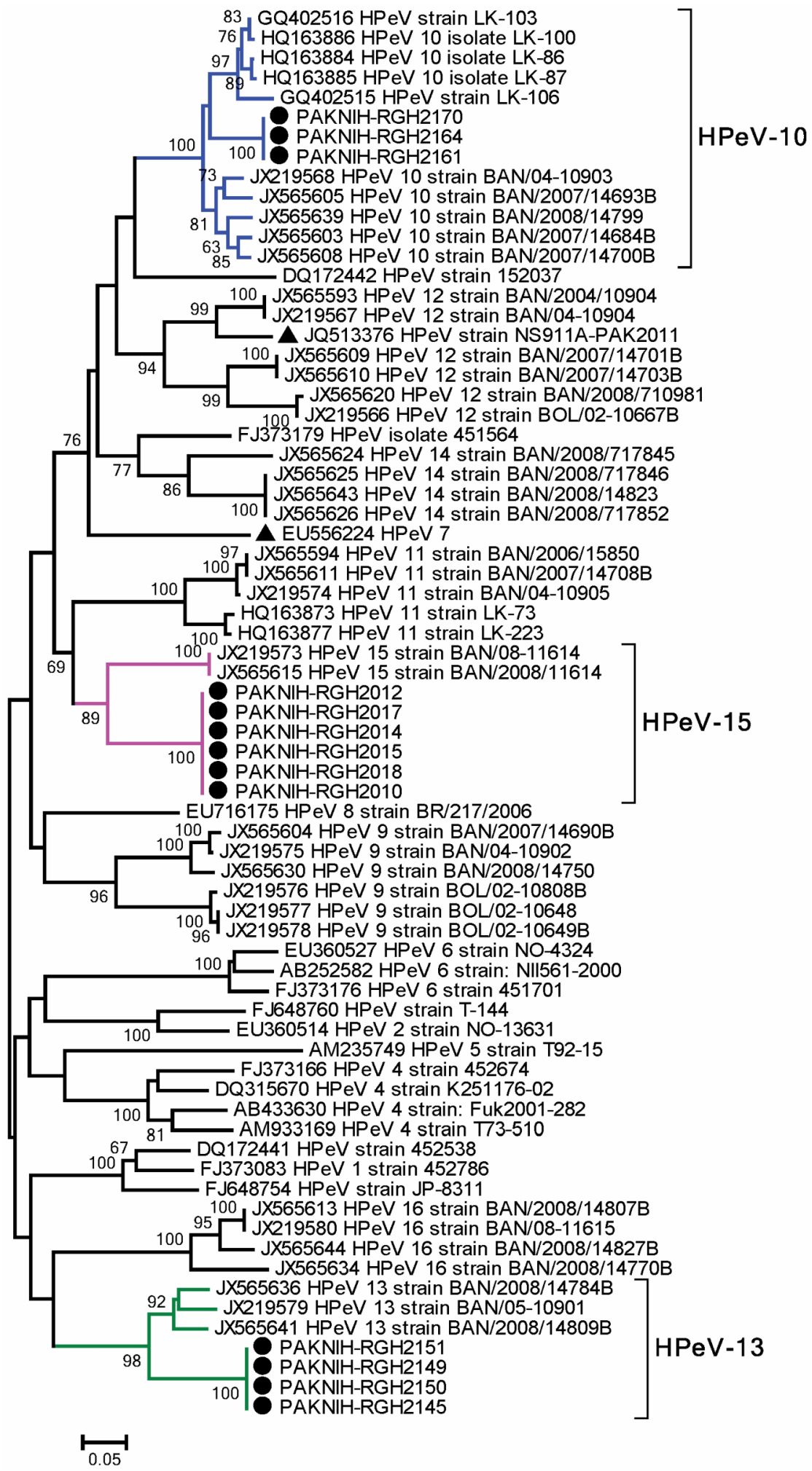

Figure 2. Phylogenetic analysis of human parechovirus strains identified in this study based on the partial VP1 region. The reference strains and closest match isolates detected through BLAST are given for genetic comparison. The phylogenetic tree with 1000 bootstrap replicates was reconstructed using neighbor joining method and the K-2P model through MEGA 4.0. Taxa with arrow head indicate the prototype strains within each serotype. Black circles indicate the strains found under this study in Pakistan. HPeV-12 and HPeV-7 previously reported from Pakistan are highlighted. doi:10.1371/journal.pone.0078377.g002 
The human parechoviruses are known to cause a variety of clinical infections including meningoencephalitis, encephalomyelitis, flaccid transient paralysis, nosocomial infection, neonatal sepsis, myocarditis, myositis, lymphadenopathy, hand-andmouth disease, rash, fever of unknown origin, influenza-like illness, Reye's syndrome, and hemolytic-uremic syndrome $[5,7,8,10,21-28]$. With their increasing clinical significance and dynamic epidemiology, the present study was designed to retrospectively screen the fecal samples kept archived at Department of Virology, National Institute of Health, Pakistan that were collected from hospitalized children with acute gastroenteritis. These samples were found negative for rotavirus antigen using the ProSpec $\mathrm{T}^{\mathrm{TM}}$ Rotavirus Microplate Assay (Oxoid Ltd., Basingstoke Hants, UK). Out of total 110, we found 26\% samples positive for $\mathrm{HPeV}$ using Real-time PCR targeting 5ÚTR. When compared, we found a relatively higher prevalence of $\mathrm{HPeVs}$ in our population than the developed countries like Germany (11.6\%) and the Netherlands $(13.4 \%)$ $[14,29]$ however, the epidemiological data from the Eastern Mediterranean countries is not available. This also indicates the improved efficiency of molecular assays for direct detection of $\mathrm{HPeVs}$ as compared to cell culture which is used to pre-amplify the viral titer but have disadvantages of selective growth thus reducing the viral diversities [30]. However, these findings require to re-assure the causal relationship of $\mathrm{HPeVs}$ in pediatric infections especially gastroenteritis through screening of matched healthy controls or antibody sero-conversion testing in the convalescent sera [31].

Majority of $\mathrm{HPeV}$ infections occur in children below 5 years of age with subclinical or mild infections $(10,33,37)$ however, in adults above 5 years of age, only minor infection with $\mathrm{HPeV}-1$ has been reported [22]. The $\mathrm{HPeV}$ infections in our population were found in children less than 4 years of age. The HPeV-13 infections were present in children between 6 months to 2 years. Infection with $\mathrm{HPeV}-15$ was found in more diverse age group including patients between 1 month to 3.4 years of age; however $\mathrm{HPeV}-10$ infections were exclusively among the children less than 1 year of age (mean age $=4$ months). Infection with $\mathrm{HPeV}-10$ and -15 within the first month of life indicates the lack of maternal protection and limited viral circulation in our population. This is in contrast to other $\mathrm{HPeV}$ genotypes where 95-99\% of neonates have been found seropositive especially against the globally prevalent HPeV-1, -2 and $-3[6,32,33]$. The extent of endemicity of new $\mathrm{HPeV}$ genotypes could not be established unless the serology based epidemiological surveys are conducted. The data also highlights that the clinical screenings for $\mathrm{HPeV}$ must be implemented especially in the pediatric units and any undiagnosed case with serious complications should be screened for $\mathrm{HPeVs}$ [34].

In Pakistan, majority of $\mathrm{HPeV}$ cases were detected during summer and post-monsoon months, however, all of the $\mathrm{HPeV}-15$ infections were found in winter; a pattern similar to HPeV-1, -3, -4 and -6 which are more dominant during fall and winter in Europe and Asia [10,30,33]. Infection of human parechoviruses usually occur during late summer (July-August) and early winter (November-December) [35] but majority of these studies are focused on the common genotypes and the data related to the most recently identified genotypes 7 to 16 is largely unknown due to very limited number of cases reported globally. In the United Kingdom, HPeV-1 and -6 circulate throughout the year without any seasonal preference [35] whereas $\mathrm{HPeV}-3$ circulation has been reported to occur as bi-annual peaks and infections with $\mathrm{HPeV}-2$ have been reported sporadically [36,37]. In Pakistan, the environmental factors may play an important role in the incidence of enteric infections during the monsoon season due to increased exposure of children to the contaminated waters. During monsoon, the rain water carrying human and animal waste pollutes the ground and surface water sources (http://www.nih. org.pk/files/Newsletter/Seasonal Awareness and Alert Letter(SAAL)24th Issue.pdf) and contribute to the outbreaks of human enteric infections [38-43]. Fecal-oral transmission of enteric infections including $\mathrm{HPeV}$ is also supported by its high viral load in the stool which may be up to 502,380 copies per ml [29]. In most of the country areas, the drinking water sources are not maintained properly, nor pre-treated, resulting to major outbreaks of enteric infections (http://en.wikipedia.org/wiki/Water_ supply_and_sanitation_in_Pakistan) [14]. Therefore, a multifocused approach including all the necessary measures ranging from proper hygiene, provision of safe drinking water and improved medical facilities must be implemented to control such infections especially in the neonates and young infants who are suffered with more severe $\mathrm{HPeV}$ complications and long-term sequelae than older children [44-46].

Since the identification of $\mathrm{HPeV}$ approx. 52 years ago in association with an outbreak of gastroenteritis [3], multiple studies have evaluated the role of $\mathrm{HPeVs}$ in gastroenteritis [35,47-49]. No direct evidence of causal relationship has yet been established however $\mathrm{HPeV}$ types 4 and 6 have been implicated as cause of diarrhea in children [34]. Among the clinical signs, diarrhea and vomiting is found to be more commonly associated with $\mathrm{HPeV}-1$ than $\mathrm{HPeV}-2$ and 3 $[6,36,50]$. In Germany, $\mathrm{HPeV}_{\mathrm{s}}$ have been found to cause lowlevel enteric infections without any serious outbreaks [29]. In Thailand, $14.6 \%$ children (6-24 months) with acute gastroenteritis were found positive for $\mathrm{HPeV}$ mainly type 1, 2, 3 and 4 [50]. In Sri Lanka, 8.3\% children (aged 2-26 months) hospitalized with gastroenteritis were found positive for $\mathrm{HPeV}$ with types 1, 3, 4, 5, 10, 11 [13]. In Japan, children aged 2 months to 15 years with acute gastroenteritis were studied and $8.1 \%$ children were infected with HPeV types 1 and 3 [51]. In a Brazilian study, $\mathrm{HPeV}$ types 1, 5, 6 and 8 were identified in $16.1 \%$ children with acute diarrhea [52]. HPeV-1, -3, -4, -5, -6 and -8 have been identified in Chinese children with gastroenteritis but no causal relationship was established [53]. Such findings suggest that $\mathrm{HPeV}$ may play a significant role in endemic cases of diarrhea among infants and younger children. Although we found $\mathrm{HPeV}$ in $24 \%$ of our patients enrolled with severe dehydrating gastroenteritis, the confirmed role of $\mathrm{HPeV}$ with such clinical complications cannot be defined and require further evidence. In $23 \%$ of our samples, HPeV-10 was identified as previously reported by Pham et al., where this type was found in two child inpatients having acute gastroenteritis at a hospital in Kandy, Sri Lanka during 2005-2006 [12] and concluded the probable role of $\mathrm{HPeV}-10$ in gastroenteritis. The epidemiological and clinical data related to HPeV-13 and 15 is not available so far.

Until 2004, only two HPeV types were known, followed by identification of 14 new types within few years [54]. Despite the continued surveillance in developed countries, the new genotypes have not been found from regions other than Asia (Pakistan, Bangladesh and Sri Lanka). Multiple genotypes of parechoviruses have been reported from Pakistan; HPeV type -7 was identified in Pakistan [11], followed by HPeV-12 [4] and genotypes-10, -13 , and -15 in this study. This may represent their localized geographical existence or the infections may go un-diagnosed due to lack of routine testing in clinical settings [55]. Similarly, the worldwide distribution of these types is still being defined. 
The methodologies adopted for $\mathrm{HPeV}$ genotyping are based on sequence analysis of the hypervariable region of VP1 with threshold of $\geq 77 \%$ and $\geq 87 \%$ nucleotide and amino acid identity respectively [20]. We found 26 samples positive through real-time PCR although the typing was successful for only 13 samples. The disadvantage of typing procedures has been extensively reported and found that the VPl assays are less sensitive than the 5UTR based real-time PGR [30,50,53,56,57] due to lower sensitivity or RNA degeneracy resulting in misdiagnosis of low-titer samples [58]. On the other hand, the genomic sequence variability and the frequent recombination between 5'UTR and the capsid-encoding region prevents $\mathrm{HPeV}$ typing through 5'UTR sequencing alone [59]. Therefore, the typing assays available to date are focused on the amplification and sequencing of VP1 gene segment. Compared to the other clinical diagnostic tools, the detection of $\mathrm{HPeV}$ infection is not straight forward and require specialized technical expertise and facilities. Although $\mathrm{HPeV}-1,3,4$ and 6 have been recovered through a variety of cell lines i.e. BSC-1, CaCo2, RD-18S, LLCMK2, Vero and HeLa [10], there is no established set of standard cell lines that can be used to isolate all $\mathrm{HPeV}$ genotypes especially the recently identified types $7-16$. The in-vitro growth adaptation of parechoviruses is usually poor and may take up to 14-18 days in addition to the variable growth characteristics of different types $[8,60,61]$ therefore, the virus isolation is not recommended for the clinical diagnosis $[11,19,52,59,61-65]$.

Similarly, serotyping of viral types require cultivation of the isolate which is practically cumbersome and fails to detect new types or variants of known types [66]. The serological assays used to detect $\mathrm{HPeV}$-specific antibody responses are usually time extensive and the lack of commercial reagents make them impractical for routine clinical use $[67,68]$. These limitations led to the development of molecular assays that initially targeted only HPeV-1 and -2 [59,62,69] and/or HPeV-1 to -6 requiring multiple sets of primers [19,29,70-73] but have now been improved to target all known genotypes and have become gold standard for the detection of $\mathrm{HPeV}$ in clinical samples [69,74]. In 2008, Nix et al., designed a real-time PCR with the detection threshold of 30 RNA copies [18] and found to be 100- to 1,000fold more sensitive than virus isolation in cell culture. Although this assay was designed on the basis of available sequences for $\mathrm{HPeV}-1$ to -6 , but our findings substantiates its vast application in the diagnostic settings targeting all 16 genotypes known so far.

In contrast to other picornaviruses, the capsid protein, VP1, of $\mathrm{HPeVs}$ is found to be poorly reactive and the predominant antigenic sites of $\mathrm{HPeV}$ have been mapped to the N-terminal region of the VP0 protein which is not antigenic in any other genera of the picornaviridae family [75]. Also, the C-terminal region of VP1 protein of $\mathrm{HPeV}$ containing arginine-glycine-aspartic acid (RGD) motif is found to be immune-reactive. The VP1 gene of $\mathrm{HPeV}-1,-2,-4,-5$ and -6 contains this motif utilized for cell-cell or cell-matrix interactions [76-79]. This motif is used by a number of picornaviruses including foot-and-mouth disease virus, echovirus 9 [80] and Coxsackievirus A9 for interaction with the host cell surface $\alpha v \beta 1$ and $\alpha_{v} \beta 3$ integrin receptors [81-85]. However, no such motif has been identified for $\mathrm{HPeV}-10,-13$ and -15 found in this study and provide evidence for the presence of an RGD-independent pathway of viral entry to host cells but no such pathway has yet been identified.

In our samples, the parechovirus genotype 15 has been found for the first time in human population after its only detection from

\section{References}

1. Hyypia T, Horsnell C, Maaronen M, Khan M, Kalkkinen N, et al. (1992) A distinct picornavirus group identified by sequence analysis. Proc Natl Acad Sci U S A 89: 8847-8851. rhesus macaques in Bangladesh [86]. However, many other $\mathrm{HPeV}$ types i.e. HPeV-1, -2, -4, -5, -12 and -14 have been reported from non-human primates (rhesus macaque) in Bangladesh and China [86,87]. Similarly, in Bolivia, pigs have been found to be infected with HPeV-4 [88]. In 2013, Reuter et al., has reported two new picornaviruses; bovine hungarovirus and ovine hungarovirus, discovered in domestic animals that contain $\mathrm{HPeV}$-like genetic markers in their 5'UTR [89]. In addition, a new picornavirus, Ljungan virus, has been identified from bank voles (Clethrionomys glareolus) in Sweden with remarkable capsid sequence homology to human parechoviruses [90]. Such a growing evidence of shared parechovirus reservoir between humans and animal species raise austere concerns of their zoonotic potential and the unidentified animal reservoir for human infections. Such findings also highlight our incomplete knowledge about the cellular tropism, virulence and cross-species transmission potential for parechoviruses [89]. Recently, in Pakistan, we have found human rotavirus G6P [1] strains that are closely resembled to bovine rotaviruses [16]. These findings highlight the risk of potential environment for the zoonotic infections in our country. Being an agricultural country, majority of Pakistani population is dependent on livestock farming which is the sole source of livelihood in many country areas. Therefore, such epidemiological studies should be extended to those country areas where animals live in close proximity to humans.

\section{Conclusion}

Our data further expand the knowledge about the prevailing $\mathrm{HPeV}$ genotypes and their clinical association in children hospitalized with acute gastroenteritis. Genotypes HPeV-10, -13 and -15 are hereby reported for the first time in our population. Considerable diversity of $\mathrm{HPeV}$ types prevail in Pakistan, therefore, large scale epidemiological and sero-surveys should be conducted to understand their clinical relevance, epidemiology and to improve preventive measures. The infections of parechoviruses in domestic and wild animals raise several concerns about their potential zoonotic risks and need further studies.

In conclusion, $\mathrm{HPeV}$ infections are quite common in our population. Pakistan is among one of the Asian countries where diarrhea associated deaths are significantly higher [91]. Despite these distressing facts, viral screenings are not performed in our country hospitals as the proper laboratory facilities are not in place due to limited resources. In addition, lack of awareness among the clinicians [92] also contributes to the undiagnosed or misdiagnosed viral infections that are usually treated with unnecessary antibiotic regimen $[19,59]$. This is in contrast to the developed settings like USA and the Netherlands where routine surveillance programs for $\mathrm{HPeVs}$ are in place for years [36]. The enormous genetic diversity and disease burden in very early age groups of our population further emphasize to prioritize the testing of $\mathrm{HPeVs}$ especially in the neonates and early-age infants.

\section{Author Contributions}

Conceived and designed the experiments: SSZZ MN. Performed the experiments: MMA AK RMS. Analyzed the data: NN MA S. Shaukat. Contributed reagents/materials/analysis tools: S.Sharif SSZZ. Wrote the paper: MMA NN SSZZ.

2. Stanway G, Hyypia T (1999) Parechoviruses. J Virol 73: 5249-5254.

3. Wigand R, Sabin AB (1961) Properties of ECHO types 22, 23 and 24 viruses. Arch Gesamte Virusforsch 11: 224-247. 
4. Alam MM, Khurshid A, Shaukat S, Sharif S, Rana MS, et al. (2012) Identification of human parechovirus genotype, $\mathrm{HPeV}-12$, in a paralytic child with diarrhea. J Clin Virol 55: 339-342.

5. Legay V, Chomel JJ, Fernandez E, Lina B, Aymard M, et al. (2002) Encephalomyelitis due to human parechovirus type 1. J Clin Virol 25: 193-195.

6. Stanway G, Joki-Korpela P, Hyypia T (2000) Human parechoviruses-biology and clinical significance. Rev Med Virol 10: 57-69.

7. Ito M, Yamashita T, Tsuzuki H, Takeda N, Sakae K (2004) Isolation and identification of a novel human parechovirus. J Gen Virol 85: 391-398.

8. Boivin G, Abed Y, Boucher FD (2005) Human parechovirus 3 and neonatal infections. Emerg Infect Dis 11: 103-105.

9. Benschop KS, Schinkel J, Luken ME, van den Broek PJ, Beersma MF, et al. (2006) Fourth human parechovirus serotype. Emerg Infect Dis 12: 1572-1575.

10. Watanabe K, Oie M, Higuchi M, Nishikawa M, Fujii M (2007) Isolation and characterization of novel human parechovirus from clinical samples. Emerg Infect Dis 13: 889-895.

11. Li L, Victoria J, Kapoor A, Naeem A, Shaukat S, et al. (2009) Genomic characterization of novel human parechovirus type. Emerg Infect Dis 15: 288291.

12. Kim Pham NT, Trinh QD, Takanashi S, Abeysekera C, Abeygunawardene A, et al. (2010) Novel human parechovirus, Sri Lanka. Emerg Infect Dis 16: 130132.

13. Pham NT, Takanashi S, Tran DN, Trinh QD, Abeysekera C, et al. (2011) Human parechovirus infection in children hospitalized with acute gastroenteritis in Sri Lanka. J Clin Microbiol 49: 364-366.

14. Benschop K, Thomas X, Serpenti C, Molenkamp R, Wolthers K (2008) High prevalence of human Parechovirus (HPeV) genotypes in the Amsterdam region and identification of specific $\mathrm{HPeV}$ variants by direct genotyping of stool samples. J Clin Microbiol 46: 3965-3970.

15. Qazi R, Sultana S, Sundar S, Warraich H, un-Nisa T, et al. (2009) Populationbased surveillance for severe rotavirus gastroenteritis in children in Karachi, Pakistan. Vaccine 27 Suppl 5: F25-30.

16. Tamim S, Hasan F, Matthijnssens J, Sharif S, Shaukat S, et al. (2013) Epidemiology and phylogenetic analysis of VP7 and VP4 genes of rotaviruses circulating in Rawalpindi, Pakistan during 2010. Infect Genet Evol 14: 161-168.

17. Alam MM, Malik SA, Shaukat S, Naeem A. Sharif S, et al. (2009) Genetic characterization of rotavirus subtypes in Pakistan-first report of G12 genotype from Pakistan under WHO-Eastern Mediterranean region. Virus Res 144: 280284.

18. Nix WA, Maher K, Johansson ES, Niklasson B, Lindberg AM, et al. (2008) Detection of all known parechoviruses by real-time PCR. J Clin Microbiol 46: 2519-2524.

19. Benschop KS, Schinkel J, Minnaar RP, Pajkrt D, Spanjerberg L, et al. (2006) Human parechovirus infections in Dutch children and the association between serotype and disease severity. Clin Infect Dis 42: 204-210.

20. Nix WA, Maher K, Pallansch MA, Oberste MS (2010) Parechovirus typing in clinical specimens by nested or semi-nested PCR coupled with sequencing. J Clin Virol 48: 202-207

21. Ehrnst A, Eriksson M (1996) Echovirus type 23 observed as a nosocomial infection in infants. Scand J Infect Dis 28: 205-206.

22. Ehrnst A, Eriksson M (1993) Epidemiological features of type 22 echovirus infection. Scand J Infect Dis 25: 275-281.

23. Figueroa JP, Ashley D, King D, Hull B (1989) An outbreak of acute flaccid paralysis in Jamaica associated with echovirus type 22. J Med Virol 29: 315-319.

24. Grist NR, Bell EJ, Assaad F (1978) Enteroviruses in human disease. Prog Med Virol 24: 114-157.

25. Koskiniemi M, Paetau R, Linnavuori K (1989) Severe encephalitis associated with disseminated echovirus 22 infection. Scand J Infect Dis 21: 463-466.

26. Maller HM, Powars DF, Horowitz RE, Portnoy B (1967) Fatal myocarditis associated with ECHO virus, type 22, infection in a child with apparent immunological deficiency. J Pediatr 71: 204-210.

27. O'Regan S, Robitaille P, Mongeau JG, McLaughlin B (1980) The hemolytic uremic syndrome associated with ECHO 22 infection. Clin Pediatr (Phila) 19: $125-127$.

28. Grist NR (1970) Echoviruses and carditis. Lancet 1: 1395.

29. Baumgarte S, de Souza Luna LK, Grywna K, Panning M, Drexler JF, et al. (2008) Prevalence, types, and RNA concentrations of human parechoviruses, including a sixth parechovirus type, in stool samples from patients with acute enteritis. J Clin Microbiol 46: 242-248.

30. Benschop K, Minnaar R, Koen G, van Eijk H, Dijkman K, et al. (2010) Detection of human enterovirus and human parechovirus $(\mathrm{HPeV})$ genotypes from clinical stool samples: polymerase chain reaction and direct molecular typing, culture characteristics, and serotyping. Diagn Microbiol Infect Dis 68: 166-173.

31. Li L, Delwart E (2011) From orphan virus to pathogen: the path to the clinical lab. Curr Opin Virol 1: 282-288.

32. Takao S, Shimazu Y, Fukuda S, Noda M, Miyazaki K (2001) Seroepidemiological study of human Parechovirus 1. Jpn J Infect Dis 54: 85-87.

33. Tauriainen S, Martiskainen M, Oikarinen S, Lonnrot M, Viskari H, et al. (2007) Human parechovirus 1 infections in young children-no association with type 1 diabetes. J Med Virol 79: 457-462.

34. Harvala H, Robertson I, Chieochansin T, McWilliam Leitch EC, Templeton K, et al. (2009) Specific association of human parechovirus type 3 with sepsis and fever in young infants, as identified by direct typing of cerebrospinal fluid samples. J Infect Dis 199: 1753-1760.

35. Harvala H, Robertson I, McWilliam Leitch EC, Benschop K, Wolthers KC, et al. (2008) Epidemiology and clinical associations of human parechovirus respiratory infections. J Clin Microbiol 46: 3446-3453.

36. van der Sanden S, de Bruin E, Vennema H, Swanink C, Koopmans M, et al. (2008) Prevalence of human parechovirus in the Netherlands in 2000 to 2007. J Clin Microbiol 46: 2884-2889.

37. Wildenbeest JG, Harvala H, Pajkrt D, Wolthers KC (2010) The need for treatment against human parechoviruses: how, why and when? Expert Rev Anti Infect Ther 8: 1417-1429.

38. Abbaszadegan M, Stewart P, LeChevallier M (1999) A strategy for detection of viruses in groundwater by PCR. Appl Environ Microbiol 65: 444-449.

39. Bosch A (1998) Human enteric viruses in the water environment: a minireview. Int Microbiol 1: 191-196.

40. Chapron CD, Ballester NA, Fontaine JH, Frades CN, Margolin AB (2000) Detection of astroviruses, enteroviruses, and adenovirus types 40 and 41 in surface waters collected and evaluated by the information collection rule and an integrated cell culture-nested PCR procedure. Appl Environ Microbiol 66: 2520-2525

41. Pianetti A, Baffone W, Citterio B, Casaroli A, Bruscolini F, et al. (2000) Presence of enteroviruses and reoviruses in the waters of the Italian coast of the Adriatic Sea. Epidemiol Infect 125: 455-462.

42. Puig M, Jofre J, Lucena F, Allard A, Wadell G, et al. (1994) Detection of adenoviruses and enteroviruses in polluted waters by nested PCR amplification. Appl Environ Microbiol 60: 2963-2970.

43. Schvoerer E, Ventura M, Dubos O, Cazaux G, Serceau R, et al. (2001) Qualitative and quantitative molecular detection of enteroviruses in water from bathing areas and from a sewage treatment plant. Res Microbiol 152: 179-186.

44. Sedmak G, Nix WA, Jentzen J, Haupt TE, Davis JP, et al. (2010) Infant deaths associated with human parechovirus infection in Wisconsin. Clin Infect Dis 50: 357-361.

45. Eyssette-Guerreau S, Boize P, Thibault M, Sarda H (2013) [Neonatal Parechovirus infection, fever, irritability and myositis]. Arch Pediatr 20: $772-$ 774 .

46. Verboon-Maciolek MA, Krediet TG, Gerards LJ, de Vries LS, Groenendaal F, et al. (2008) Severe neonatal parechovirus infection and similarity with enterovirus infection. Pediatr Infect Dis J 27: 241-245.

47. Chhabra P, Payne DC, Szilagyi PG, Edwards KM, Staat MA, et al. (2013) Etiology of Viral Gastroenteritis in Children $<5$ Years of Age in the United States, 2008-2009. J Infect Dis.

48. Ghazi F, Ataei Z, Dabirmanesh B (2012) Molecular detection of human parechovirus type 1 in stool samples from children with diarrhea. Int J Infect Dis 16: e673-676.

49. Tapia G, Cinek O, Witso E, Kulich M, Rasmussen T, et al. (2008) Longitudinal observation of parechovirus in stool samples from Norwegian infants. J Med Virol 80: 1835-1842.

50. Pham NT, Trinh QD, Khamrin P, Maneekarn N, Shimizu H, et al. (2010) Diversity of human parechoviruses isolated from stool samples collected from Thai children with acute gastroenteritis. J Clin Microbiol 48: 115-119.

51. Pham NT, Chan-It W, Khamrin P, Nishimura S, Kikuta H, et al. (2011) Detection of human parechovirus in stool samples collected from children with acute gastroenteritis in Japan during 2007-2008. J Med Virol 83: 331-336.

52. Drexler JF, Grywna K, Stocker A, Almeida PS, Medrado-Ribeiro TC, et al. (2009) Novel human parechovirus from Brazil. Emerg Infect Dis 15: 310-313.

53. Zhang DL, Jin Y, Li DD, Cheng WX, Xu ZQ, et al. (2011) Prevalence of human parechovirus in Chinese children hospitalized for acute gastroenteritis. Clin Microbiol Infect 17: 1563-1569.

54. Romero JR, Selvarangan R (2011) The human Parechoviruses: an overview. Adv Pediatr 58: 65-85.

55. Harvala H, Wolthers KC, Simmonds P (2010) Parechoviruses in children: understanding a new infection. Curr Opin Infect Dis 23: 224-230.

56. Zhang DL, Zhang Q, Li DD, Cheng WX, Xu ZQ, et al. (2009) Detection of human parechovirus in children hospitalized for acute gastroenteritis]. Zhonghua Shi Yan He Lin Chuang Bing Du Xue Za Zhi 23: 112-114.

57. Ito M, Yamashita T, Tsuzuki H, Kabashima Y, Hasegawa A, et al. (2010) Detection of human parechoviruses from clinical stool samples in Aichi, Japan. J Clin Microbiol 48: 2683-2688.

58. Khetsuriani N, Lamonte-Fowlkes A, Oberst S, Pallansch MA (2006) Enterovirus surveillance-United States, 1970-2005. MMWR Surveill Summ 55: 1-20.

59. Harvala H, Simmonds P (2009) Human parechoviruses: biology, epidemiology and clinical significance. J Clin Virol 45: 1-9.

60. Shoja ZO, Tabatabie H, Shahmahmoudi S, Nategh R (2007) Comparison of cell culture with RT-PCR for enterovirus detection in stool specimens from patients with acute flaccid paralysis. J Clin Lab Anal 21: 232-236.

61. van Doornum GJ, Schutten M, Voermans J, Guldemeester GJ, Niesters HG (2007) Development and implementation of real-time nucleic acid amplification for the detection of enterovirus infections in comparison to rapid culture of various clinical specimens. J Med Virol 79: 1868-1876.

62. Noordhoek GT, WeelJF, Poelstra E, Hooghiemstra M, Brandenburg AH (2008) Clinical validation of a new real-time PCR assay for detection of enteroviruses and parechoviruses, and implications for diagnostic procedures. J Clin Virol 41: 75-80. 
63. Iturriza-Gomara M, Megson B, Gray J (2006) Molecular detection and characterization of human enteroviruses directly from clinical samples using RT-PCR and DNA sequencing. J Med Virol 78: 243-253.

64. Shoja ZO, Tabatabai H, Sarijloo M, Shahmahmoodi S, Azad TM, et al. (2007) Detection of enteroviruses by reverse-transcriptase polymerase chain reaction in cell culture negative stool specimens of patients with acute flaccid paralysis. J Virol Methods 142: 95-97.

65. Terletskaia-Ladwig E, Meier S, Hahn R, Leinmuller M, Schneider F, et al. (2008) A convenient rapid culture assay for the detection of enteroviruses in clinical samples: comparison with conventional cell culture and RT-PCR. J Med Microbiol 57: 1000-1006.

66. Balows A HW, Lennette E, Eds. (1988) The enteroviruses (polioviruses, coxsackieviruses, echoviruses. In: JG K, editor. Laboratory diagnosis of infectious diseases Principles and practice. New York: Springer-Verlag. pp. $692-722$.

67. Abed Y, Wolf D, Dagan R, Boivin G (2007) Development of a serological assay based on a synthetic peptide selected from the VP0 capsid protein for detection of human parechoviruses. J Clin Microbiol 45: 2037-2039.

68. Alho A, Marttila J, Ilonen J, Hyypia T (2003) Diagnostic potential of parechovirus capsid proteins. J Clin Microbiol 41: 2294-2299.

69. Benschop K, Molenkamp R, van der Ham A, Wolthers K, Beld M (2008) Rapid detection of human parechoviruses in clinical samples by real-time PCR. J Clin Virol 41: 69-74.

70. Corless CE, Guiver M, Borrow R, Edwards-Jones V, Fox AJ, et al. (2002) Development and evaluation of a 'real-time' RT-PCR for the detection of enterovirus and parechovirus RNA in CSF and throat swab samples. J Med Virol 67: 555-562.

71. Donoso Mantke O, Kallies R, Niklasson B, Nitsche A, Niedrig M (2007) A new quantitative real-time reverse transcriptase PCR assay and melting curve analysis for detection and genotyping of Ljungan virus strains. J Virol Methods 141: 71-77.

72. Jokela P, Joki-Korpela P, Maaronen M, Glumoff V, Hyypia T (2005) Detection of human picornaviruses by multiplex reverse transcription-PCR and liquid hybridization. J Clin Microbiol 43: 1239-1245.

73. Oberste MS, Maher K, Pallansch MA (1999) Specific detection of echoviruses 22 and 23 in cell culture supernatants by RT-PCR. J Med Virol 58: 178-181.

74. Abed Y, Boivin G (2006) Human parechovirus types 1, 2 and 3 infections in Canada. Emerg Infect Dis 12: 969-975.

75. Joki-Korpela P, Roivainen M, Lankinen H, Poyry T, Hyypia T (2000) Antigenic properties of human parechovirus 1. J Gen Virol 81: 1709-1718.

76. Ruoslahti E, Pierschbacher MD (1987) New perspectives in cell adhesion: RGD and integrins. Science 238: 491-497.

77. Al-Sunaidi M, Williams CH, Hughes PJ, Schnurr DP, Stanway G (2007) Analysis of a new human parechovirus allows the definition of parechovirus types and the identification of RNA structural domains. J Virol 81: 1013-1021.
78. Boonyakiat Y, Hughes PJ, Ghazi F, Stanway G (2001) Arginine-glycine-aspartic acid motif is critical for human parechovirus 1 entry. J Virol 75: 10000-10004.

79. Stanway G, Kalkkinen N, Roivainen M, Ghazi F, Khan M, et al. (1994) Molecular and biological characteristics of echovirus 22, a representative of a new picornavirus group. J Virol 68: 8232-8238.

80. Zimmermann H, Eggers HJ, Nelsen-Salz B (1996) Molecular cloning and sequence determination of the complete genome of the virulent echovirus 9 strain barty. Virus Genes 12: 149-154.

81. Chang KH, Auvinen P, Hyypia T, Stanway G (1989) The nucleotide sequence of coxsackievirus A9; implications for receptor binding and enterovirus classification. J Gen Virol 70 (Pt 12): 3269-3280.

82. Chang KH, Day C, Walker J, Hyypia T, Stanway G (1992) The nucleotide sequences of wild-type coxsackievirus A9 strains imply that an RGD motif in VP1 is functionally significant. J Gen Virol 73 (Pt 3): 621-626.

83. Fox G, Parry NR, Barnett PV, McGinn B, Rowlands DJ, et al. (1989) The cell attachment site on foot-and-mouth disease virus includes the amino acid sequence RGD (arginine-glycine-aspartic acid). J Gen Virol 70 (Pt 3): 625637.

84. Hyypia T, Stanway G (1993) Biology of coxsackie A viruses. Adv Virus Res 42: 343-373.

85. Hyypia T, Hovi T, Knowles NJ, Stanway G (1997) Classification of enteroviruses based on molecular and biological properties. J Gen Virol 78 (Pt 1): $1-11$.

86. Oberste MS, Feeroz MM, Maher K, Nix WA, Engel GA, et al. (2013) Characterizing the picornavirus landscape among synanthropic nonhuman primates in Bangladesh, 2007 to 2008. J Virol 87: 558-571.

87. Shan TL, Wang CM, Cui L, Delwart E, Yuan CL, et al. (2010) Human parechovirus infections in monkeys with diarrhea, China. Emerg Infect Dis 16: 1168-1169.

88. Nix WA, Khetsuriani N, Penaranda S, Maher K, Venczel L, et al. (2013) Diversity of picornaviruses in rural Bolivia. J Gen Virol.

89. Reuter G, Pankovics P, Knowles NJ, Boros A (2012) Two closely related novel picornaviruses in cattle and sheep in Hungary from 2008 to 2009, proposed as members of a new genus in the family Picornaviridae. J Virol 86: 13295-13302.

90. Tolf C, Gullberg M, Johansson ES, Tesh RB, Andersson B, et al. (2009) Molecular characterization of a novel Ljungan virus (Parechovirus; Picornaviridae) reveals a fourth genotype and indicates ancestral recombination. J Gen Virol 90: 843-853.

91. Kawai K, O'Brien MA, Goveia MG, Mast TC, El Khoury AC (2012) Burden of rotavirus gastroenteritis and distribution of rotavirus strains in Asia: a systematic review. Vaccine 30: 1244-1254.

92. Landry ML (2010) The molecular diagnosis of parechovirus infection: has the time come? Clin Infect Dis 50: 362-363. 